\title{
The q-Stirling numbers of the second kind and its applica- tions
}

\author{
Min-Soo Kim ${ }^{\mathrm{a}}$, Daeyeoul Kim ${ }^{\mathrm{b}, *}$ \\ ${ }^{a}$ Division of Mathematics, Science, and Computers, Kyungnam University, 7(Woryeong-dong) kyungnamdaehak-ro, Masanhappo-gu, \\ Changwon-si, Gyeongsangnam-do 51767, Republic of Korea. \\ ${ }^{b}$ Department of Mathematics and Institute of Pure and Applied Mathematics, Chonbuk National University, Jeonju-si 54896, Republic of \\ Korea.
}

Communicated by S.-H. Rim

\begin{abstract}
The study of q-Stirling numbers of the second kind began with Carlitz [L. Carlitz, Duke Math. J., 15 (1948), 987-1000] in 1948. Following Carlitz, we derive some identities and relations related to q-Stirling numbers of the second kind which appear to be either new or else new ways of expressing older ideas more comprehensively.
\end{abstract}

Keywords: q-Stirling numbers of the second kind, q-factorial.

2010 MSC: 05A40, 11A25.

(C)2018 All rights reserved.

\section{Introduction}

In mathematics, the Stirling numbers of second kind have been studied from many diverse viewpoints. Originally, Stirling numbers of the second kind $S(n, m)$ are equal to the number of partitions of the set $\{1,2, \ldots, n\}$ into $m$ non-empty disjoint sets. They have the important recurrence relation

$$
S(n, m)=S(n-1, m-1)+m S(n-1, m)
$$

with the conditions $S(n, 0)=\delta_{n, 0}$ and $S(0, m)=\delta_{0, m}$ for all $n, m \in \mathbb{N} \cup\{0\}$. Here,

$$
\delta_{m, m}= \begin{cases}1, & \text { if } n=m, \\ 0, & \text { otherwise }\end{cases}
$$

A proof of (1.1) using finite differences is presented in [12]. It is known that, for $n \geqslant m \geqslant 0, S(n, m)$ can

\footnotetext{
${ }^{*}$ Corresponding author

Email addresses: mskim@kyungnam.ac.kr (Min-Soo Kim), kdaeyeoul@jbnu.ac.kr (Daeyeoul Kim)

doi: $10.22436 /$ jnsa.011.08.04
}

Received: 2018-03-31 Revised: 2018-05-01 Accepted: 2018-05-03 
be computed by the explicit formula (also discussed in [12])

$$
S(n, m)=\frac{1}{m !} \sum_{j=0}^{m}(-1)^{m-j}\left(\begin{array}{c}
m \\
j
\end{array}\right) j^{n},
$$

(see $[1,13,16,19] ;$ and the references cited therein).

Gould [12] discussed some combinatorial identities related to (1.2) via finite differences. A combinatorial proof of (1.2) based on the combinatorial definition of $S(n, m)$ can be found in [7, pp. 204-205]; a proof based on finite differences is given in [16, p. 169; see also 177-178 and 189-190].

Simsek [25] constructed new generating functions for the generalized $\lambda$-Stirling type numbers of the second kind, generalized array type polynomials, generalized Eulerian type polynomials and numbers. He derived some new identities, various functional equations and differential equations using these generating functions. Also, Simsek [24] obtained some combinatorial sums and identities including the Bernoulli numbers and polynomials, the Stirling numbers of the second kind and some relations by using the Bernstein basis functions and Bernstein operator with their integral.

A viewpoint of Carlitz [3], motivated by the counting problem for Abelian groups, is to study the Stirling numbers as specializations of the q-String numbers. Now, we briefly summarize some basic properties of $q$-calculus. Let $n \in \mathbb{N}$ and $q \in(0,1)$. For $n$ an integer, the $q$-integer $[n]_{q}$ and $q$-factorial $[n]_{q}$ ! are respectively defined by

$$
[n]_{q}=\frac{1-q^{n}}{1-q}=1+q+\cdots+q^{n-1}, \quad[n]_{q} != \begin{cases}1, & \text { if } n=0, \\ {[n]_{q}[n-1]_{q} \cdots[1]_{q},} & \text { if } n \in \mathbb{N} .\end{cases}
$$

The Gaussian binomial coefficient is defined by

$$
\left(\begin{array}{l}
n \\
k
\end{array}\right)_{q}=\frac{[n]_{q} !}{[n-k]_{q} ![k]_{q} !}=\left(\begin{array}{c}
n \\
n-k
\end{array}\right)_{q}, \quad 1 \leqslant k \leqslant n
$$

with $\left(\begin{array}{l}n \\ 0\end{array}\right)_{q}=1$ and $\left(\begin{array}{l}n \\ k\end{array}\right)_{q}=0$ for $n<k$.

By a $q$-number $[x]_{q}$ we mean

$$
[x]_{\mathrm{q}}=\frac{1-\mathrm{q}^{\mathrm{x}}}{1-\mathrm{q}}
$$

which is well-defined for real $x$. If $x$ is a natural number $n$, it would seem appropriate to speak of $[n]_{q}$ as a q-natural number. By a q-binomial coefficient we shall of course mean

$$
\left(\begin{array}{l}
x \\
k
\end{array}\right)_{q}=\frac{[x]_{\mathrm{q}}[x-1]_{\mathrm{q}} \cdots[x-k+1]_{\mathrm{q}}}{[k]_{\mathrm{q}} !}=\prod_{i=1}^{k} \frac{1-\mathrm{q}^{\mathrm{x}-\mathrm{i}+1}}{1-\mathrm{q}^{i}} .
$$

Ward [30] has remarked about the lack of uniqueness and trouble which sometimes arises because of the presence of powers of $\mathrm{q}$ in such pseudo-isomorphisms as

$$
[a+b]_{q}=q^{b}[a]_{q}+[b]_{q}=[a]_{q}+q^{a}[b]_{q} .
$$

Thus we shall also need the following easily verified relations:

$$
\mathrm{q}^{\mathrm{n}}[\mathrm{x}-\mathrm{n}]_{\mathrm{q}}=[\mathrm{x}]_{\mathrm{q}}-[\mathrm{n}]_{\mathrm{q}}, \quad[-x]_{\mathrm{q}}=-\mathrm{q}^{-\mathrm{x}}[\mathrm{x}]_{\mathrm{q}}, \quad\left(\begin{array}{l}
x \\
\mathrm{n}
\end{array}\right)_{\mathrm{q}}=\frac{[x]_{\mathrm{q}}}{[n]_{\mathrm{q}}}\left(\begin{array}{c}
x-1 \\
n-1
\end{array}\right)_{\mathrm{q}} .
$$

We will use a well known q-analogue of the Stirling numbers of the second kind that goes back at least to Carlitz ([3]; see also [21, 29]). We have the q-Stirling numbers of the second kind defined by Carlitz as numbers $a_{n, k}$ such that

$$
[x]_{\mathrm{q}}^{\mathrm{n}}=\sum_{k=0}^{n} \mathrm{q}^{\left(\begin{array}{l}
k \\
2
\end{array}\right)}\left(\begin{array}{l}
x \\
k
\end{array}\right)_{\mathrm{q}}[k]_{\mathrm{q}} ! \mathrm{a}_{\mathrm{n}, \mathrm{k}}
$$


from which Carlitz found

$$
a_{n, k}=\frac{q^{-\left(\begin{array}{l}
k \\
2
\end{array}\right)}}{[k]_{q} !} \sum_{j=0}^{k}(-1)^{j} q^{\left(\begin{array}{l}
j \\
2
\end{array}\right)}\left(\begin{array}{l}
k \\
j
\end{array}\right)_{q}[k-j]_{q}^{n} .
$$

They arose in connection with a problem of abelian group in [4]. Note that this expression reduces when $q \rightarrow 1$ to (1.2) above.

The q-analog of Stirling numbers have been studied over the years by Carlitz [3, 4] and Gould [13]. Later, many important properties of q-Stirling numbers can be found in Milne [21] or Cigler [6]. Sagan [23] considered various arithmetic properties of q-Stirling numbers of both kinds. Zeng [31] gave continued fraction expansions for the ordinary generating functions of the q-Stirling numbers of both kinds. In [22], Ozden et al. studied some identities and relations related to q-Bernoulli numbers and polynomials and q-Stirling numbers of the second kind.

Our main aim is to find some identities and relations related to the q-Stirling numbers of the second kind. The main results of this paper may be stated in Section 3.

\section{Preliminaries}

We consider a Carlitz's q-difference operational argument due to Kim and Son [17]. Motivated by such applications, we present q-analogues of Newton series by using q-difference operator (see (2.1) below).

If the constant difference between successive values of $x$ is $h$, so that the general value of $x$ is $x_{k}=$ $x_{0}+k h$ with $k \in \mathbb{Z}$, and the corresponding functional value is $f\left(x_{k}\right)=f\left(x_{0}+k h\right)=f_{k}$. Let $E_{h}$ be the translating operator defined by

$$
E_{h}\left(f_{k}\right)=f\left(x_{k}+h\right)=f\left(x_{k+1}\right) .
$$

Applying $E_{h}$ again increases the argument of $f$ by $h$, i.e.,

$$
E_{h}^{2} f\left(x_{k}\right)=E_{h}\left(E_{h} f\left(x_{k}\right)\right)=f\left(x_{k}+2 h\right)=f\left(x_{k+2}\right)=f_{k+2}
$$

and generally $E_{h}^{r} f\left(x_{k}\right)=f_{k+r}$ for $r \in \mathbb{N}$.

Now we consider the Carlitz's q-difference operator

$$
\triangle_{q, h}^{n}= \begin{cases}I, & \text { if } n=0 \\ \left(E_{h}-q^{n-1}\right) \cdots\left(E_{h}-q\right)\left(E_{h}-I\right), & \text { if } n \geqslant 1\end{cases}
$$

(see $[3,8,17])$. q-difference operator has very interesting property. For example in [17],

$$
\triangle_{q, h}^{n} f_{k}=(-1)^{n} q^{\left(\begin{array}{c}
n \\
2
\end{array}\right)} \sum_{i=0}^{n}(-1)^{i}\left(\begin{array}{l}
n \\
i
\end{array}\right) f_{q} f_{k+i} q^{i(i-2 n+1) / 2},
$$

where $n \in \mathbb{N}$. Specifically, taking $k=0$ and $n \in \mathbb{N}$, we have

$$
\triangle_{q, h}^{n} f_{0}=(-1)^{n} q^{\left(\begin{array}{c}
n \\
2
\end{array}\right)} \sum_{i=0}^{n}(-1)^{i}\left(\begin{array}{l}
n \\
i
\end{array}\right)_{q} f_{i} q^{i(i-2 n+1) / 2},
$$

which can be expressed as

$$
\triangle_{q, h}^{n} f(x)=\sum_{i=0}^{n}(-1)^{n-i} q^{\left(\begin{array}{c}
n-i \\
2
\end{array}\right)}\left(\begin{array}{c}
n \\
i
\end{array}\right)_{q} f(x+i h) .
$$

When $h=1$ in (2.1), we use the notation

$$
\triangle_{\mathrm{q}}^{\mathrm{n}}=\triangle_{\mathrm{q}, 1}^{\mathrm{n}}
$$


As convention, define $\triangle_{\mathrm{q}}^{0}=\mathrm{I}$ (the identity map). In particular, with $x=0$,

$$
\triangle_{q}^{n} f(0)=\sum_{i=0}^{n}(-1)^{n-i} q^{\left(\begin{array}{c}
n-i \\
2
\end{array}\right)}\left(\begin{array}{l}
n \\
i
\end{array}\right)_{q} f(i) .
$$

Using the binomial expansion, we arrive at the representation

$$
f_{k}=\sum_{i=0}^{k}\left(\begin{array}{l}
k \\
i
\end{array}\right)_{q} \triangle_{q, h}^{i} f_{0}
$$

The q-factorial polynomials are given

$$
[x]_{\mathfrak{q}}^{(\underline{m})}= \begin{cases}1 & \text { if } m=0, \\ {[x]_{\mathrm{q}}[x-1]_{\mathrm{q}} \cdots[x-m+1]_{\mathrm{q}},} & \text { if } m \geqslant 1\end{cases}
$$

(see $[3,15,17])$. In view of formula (2.5), it is a matter of some interest to be able to express an arbitrary function $f_{q}(x)$ in terms of the q-factorial polynomial. That is,

$$
f_{q}(x):=\sum_{m=0}^{\infty} a_{m}[x]_{q}^{(\underline{m})}=a_{0}+a_{1}[x]_{q}^{(1)}+a_{2}[x]_{q}^{(2)}+a_{3}[x]_{q}^{(3)}+\cdots .
$$

We suppose that $a_{0}=f_{q}(0)$. By (2.1) and (2.5)

$$
\triangle_{q} f_{q}(x)=-q^{x+1}\left([-1]_{q} a_{1}+[-2]_{q} a_{2}[x]_{q}^{(1)}+\cdots+[-m]_{q} a_{m}[x]_{q}^{(m-1)}+\cdots\right) .
$$

If we set $x=0$ in this expression, $[1]_{q} a_{1}=-q[-1]_{q} a_{1}=\triangle_{q} f_{q}(0)$. Similarly, we obtain $[2]_{q} ! a_{2}=$ $\triangle_{\mathrm{q}}^{2} \mathrm{f}_{\mathrm{q}}(0)$ and

where $\mathrm{m}=0,1,2, \ldots$

$$
a_{m}=\frac{\triangle_{q}^{m} f_{q}(0)}{[m]_{q} !}
$$

Therefore, we immediately have the following theorem.

Theorem 2.1. Let $\mathrm{f}_{\mathrm{q}}(\mathrm{x})$ be an arbitrary function in $\mathrm{q}$-factorial polynomials. Then

$$
\mathrm{f}_{\mathrm{q}}(\mathrm{x})=\sum_{\mathrm{m}=0}^{\infty} \frac{\triangle_{\mathrm{q}}^{\mathrm{m}} \mathrm{f}_{\mathrm{q}}(0)}{[\mathrm{m}]_{\mathrm{q}} !}[\mathrm{x}]_{\mathrm{q}}^{(\mathrm{m})},
$$

which obviously resembles the Newton series of $\mathrm{f}_{\mathbf{q}}(\mathrm{x})$ in terms of the basis $\left\{[\mathrm{x}]_{\mathrm{q}}^{(\mathrm{m})}: \mathrm{m} \in \mathbb{N}_{0}\right.$.

Remark 2.2. When we set $q \rightarrow 1$ in Theorem 2.1, we obtain an expansion of a function, say $f$, in terms of difference polynomials, $(x)^{\underline{0}}=1,(x)^{\underline{1}}=x,(x)^{\underline{2}}=x(x-1)$, and in general $(x)^{\underline{m}}=x(x-1) \cdots(x-m+1)$, that is,

$$
f(x)=\sum_{m=0}^{\infty} \frac{\triangle^{m} f(0)}{m !}(x)^{m},
$$

which is Newton series. Here, the expression $\triangle f(x)=f(x+1)-f(x)$ is the forward difference. In this way a Newton series resembles a Taylor series, which is an expansion of $f$ in terms of another basis, the power polynomials $p_{k}(x)=x^{k}$ for $k=0,1, \ldots$ (see [1]).

\section{Main results}

In this section we study three natural q-analogs of Stirling numbers of the second kind (see Propostion 3.1 below). We note particularly results Proposition 3.1 (2), Theorem 3.6, Theorem 3.7, Corollary 3.8, Theorem 3.9, and Theorem 3.10 which appear to be either new or else new ways of expressing older ideas more comprehensively. 
For example, the q-Stirling numbers of the second kind $S_{q}(n, m)$ which are defined by Carlitz $[3,(3.1)]$ as

$$
[x]_{\mathrm{q}}^{\mathrm{n}}=\sum_{\mathrm{m}=0}^{\mathrm{n}} \mathrm{q}^{\left(\begin{array}{c}
\mathrm{m} \\
2
\end{array}\right)} S_{\mathrm{q}}(\mathrm{n}, \mathrm{m})[\mathrm{x}]_{\mathrm{q}}^{(\mathrm{m})} .
$$

In fact, Carlitz gave the corresponding expression for $a_{n, m}$ in (1.5). Note that for $q \rightarrow 1$, the $q$-Stirling numbers $S_{q}(n, m)$, reduces to the well known Stirling numbers of the second kind.

The expression (3.1) may be written as

$$
\sum_{m=0}^{n+1} q^{\left(\begin{array}{c}
m \\
2
\end{array}\right)} S_{q}(n+1, m)[x]_{q}^{(\underline{m})}=[x]_{q}^{n+1}=\left(\sum_{m=0}^{n} q^{\left(\begin{array}{c}
m \\
2
\end{array}\right)} S_{q}(n, m)[x]_{q}^{\left(\frac{m}{m}\right)}\right)[x]_{q} .
$$

So putting $[x]_{q}=\left([x-m]_{q} q^{m}+[m]_{q}\right)$ in (3.2), we have

$$
\begin{aligned}
{[x]_{\mathrm{q}}^{\mathrm{n}+1} } & =\sum_{m=0}^{n} q^{\left(\begin{array}{c}
m \\
2
\end{array}\right)} S_{q}(n, m)[x]_{q}^{\left(\frac{m}{q}\right)}\left([x-m]_{q} q^{m}+[m]_{q}\right) \\
& =\sum_{m=0}^{n} q^{\left(\begin{array}{c}
m \\
2
\end{array}\right)+m} S_{q}(n, m)[x]_{q}^{\left(\frac{m}{q}+1\right)}+[m]_{q} \sum_{m=0}^{n} q^{\left(\begin{array}{c}
m \\
2
\end{array}\right)} S_{q}(n, m)[x]_{q}^{\left(\frac{m}{q}\right)} \\
& =\sum_{m=0}^{n+1}\left(q^{\left(\begin{array}{c}
m-1 \\
2
\end{array}\right)+m-1} S_{q}(n, m-1)+q^{\left(\begin{array}{c}
m \\
2
\end{array}\right)}[m]_{q} S_{q}(n, m)\right)[x]_{q}^{(m)} .
\end{aligned}
$$

To prove the next-to-last equality, use $S_{q}(0, m)=\delta_{0 m}$ and $S_{q}(n, 0)=\delta_{n 0}$. From (3.2) and (3.3) it is clear that $S_{q}(n, m)$ satisfies the recurrence relation

$$
S_{\mathrm{q}}(\mathrm{n}+1, \mathrm{~m})=\mathrm{S}_{\mathrm{q}}(\mathrm{n}, \mathrm{m}-1)+[\mathrm{m}]_{\mathrm{q}} \mathrm{S}_{\mathrm{q}}(\mathrm{n}, \mathrm{m})
$$

(see $[2,(2.1)],[3,(3.2)]$, and $[21,(1.17)])$. The $S_{q}(n, m)$ themselves are not new, they have been considered by Milne in [21], who gave them a combinatorial interpretation in terms of partitions. They are also closely related to the q-Stirling numbers of second kind introduced by Gould in [13]. Just set

$$
y_{m ; q}(t)=\sum_{n=0}^{\infty} S_{q}(n, m) \frac{t^{n}}{n !} .
$$

It is not hard to see that

$$
\frac{d}{d t} y_{m ; q}(t)-[m]_{q} y_{m ; q}(t)=y_{m-1 ; q}(t)
$$

by (3.4), where $m=1,2, \ldots$, and $y_{m ; q}(0)=0$ for $m \geqslant 1$, and $y_{0 ; q}(t)=1$.

We use Theorem 2.1 now to compute the q-Stirling numbers $S_{q}(n, m)$ in equation (3.1). Take $f_{q}(x)=$ $[x]_{\mathrm{q}}^{n}$ in Theorem 2.1 to obtain

$$
[x]_{\mathrm{q}}^{\mathrm{n}}=\sum_{\mathrm{m}=0}^{\infty}\left\{\left.\frac{1}{[\mathrm{~m}]_{\mathrm{q}} !} \triangle_{\mathrm{q}}^{\mathrm{m}}[\mathrm{x}]_{\mathrm{q}}^{\mathrm{n}}\right|_{\mathrm{x}=0}\right\}[\mathrm{x}]_{\mathrm{q}}^{(\mathrm{m})} .
$$

A simple computation shows that $\triangle_{q} q^{i x}=\left(q^{i}-1\right) q^{i x}, i \geqslant 0$ and so

$$
\triangle_{q}[x]_{q}^{n}=\triangle_{q}\left((q-1)^{-n} \sum_{i=0}^{n}\left(\begin{array}{c}
n \\
i
\end{array}\right)(-1)^{n-i} q^{i x}\right)=(q-1)^{-n} \sum_{i=0}^{n}\left(\begin{array}{c}
n \\
i
\end{array}\right)(-1)^{n-i}\left(q^{i}-1\right) q^{i x},
$$

which yields $(q-1)^{-n} \sum_{i=1}^{n}\left(\begin{array}{c}n \\ i\end{array}\right)(-1)^{n-i}\left(q^{i}-1\right)=\left.\triangle_{q}[x]_{q}^{n}\right|_{x=0}$. Also,

$$
\triangle_{q}^{2}[x]_{q}^{n}=(q-1)^{-n} \sum_{i=0}^{n}\left(\begin{array}{l}
n \\
i
\end{array}\right)(-1)^{n-i}\left(q^{i}-1\right)\left(q^{i}-q\right) q^{i x}
$$


and $(q-1)^{-n} \sum_{i=2}^{n}\left(\begin{array}{c}n \\ i\end{array}\right)(-1)^{n-i}\left(q^{i}-1\right)\left(q^{i}-q\right)=\left.\triangle_{q}^{2}[x]_{q}^{n}\right|_{x=0}$.

Continuing this way, the general formula is

$$
(q-1)^{-n} \sum_{i=m}^{n}\left(\begin{array}{l}
n \\
i
\end{array}\right)(-1)^{n-i}\left(q^{i}-1\right) \cdots\left(q^{i}-q^{m-1}\right)=\left.\triangle_{q}^{m}[x]_{q}^{n}\right|_{x=0} .
$$

Thus (3.5) becomes

$$
\begin{aligned}
{[x]_{q}^{n} } & \left.=\sum_{m=0}^{n}\left\{\frac{(q-1)^{-n}}{[m]_{q} !} \sum_{i=m}^{n}\left(\begin{array}{c}
n \\
i
\end{array}\right)(-1)^{n-i}\left(q^{i}-1\right) \cdots\left(q^{i}-q^{m-1}\right)\right\}[x]_{q}^{(m)}\right) \\
& =\sum_{m=0}^{n}\left\{(q-1)^{m-n} q^{\left(\begin{array}{c}
m \\
2
\end{array}\right)} \sum_{i=m}^{n}(-1)^{n-i}\left(\begin{array}{c}
n \\
i
\end{array}\right)\left(\begin{array}{c}
i \\
m
\end{array}\right)_{q}\right\}[x]_{q}^{(m)},
\end{aligned}
$$

since $\left.\triangle_{q}^{m}[x]_{q}^{n}\right|_{x=0}=0$ for $n<m$ and

$$
(q-1)^{m} q^{\left(\begin{array}{c}
m \\
2
\end{array}\right)}\left(\begin{array}{c}
i \\
m
\end{array}\right)_{q}[m]_{q} !=\left(q^{i}-1\right) \cdots\left(q^{i}-q^{m-1}\right) .
$$

In view of (2.4), take $f_{q}(x)=[x]_{q}^{n}$ in Theorem 2.1 to obtain

$$
[x]_{\mathrm{q}}^{n}=\sum_{m=0}^{\infty}\left\{\frac{1}{[m]_{\mathrm{q}} !} \sum_{i=0}^{m}(-1)^{m-i} q^{(m-i)}\left(\begin{array}{c}
m \\
i
\end{array}\right)_{q}[i]_{q}^{n}\right\}[x]_{q}^{(m)} .
$$

Note that (3.9) is valid for all non-negative $m, n$; in particular then the right member vanishes for $n<m$. By (3.1), (3.5), and (3.7) we prove the following result.

Proposition 3.1. Let the $\mathrm{q}$-Stirling numbers $S_{\mathrm{q}}(\mathrm{n}, \mathrm{m})$ be defined by equation (3.1). The followings are equivalent:

1. $S_{\mathrm{q}}(\mathrm{n}, \mathrm{m})=\left.\frac{\mathrm{q}^{-\left(\begin{array}{c}\mathrm{m} \\ 2\end{array}\right)}}{[\mathrm{m}]_{\mathrm{q}} !} \triangle_{\mathrm{q}}^{\mathrm{m}}[\mathrm{x}]_{\mathrm{q}}^{\mathrm{n}}\right|_{\mathrm{x}=0}$;

2. $S_{q}(n, m)=(q-1)^{m-n} \sum_{i=m}^{n}(-1)^{n-i}\left(\begin{array}{c}n \\ i\end{array}\right)\left(\begin{array}{c}i \\ m\end{array}\right) q$;

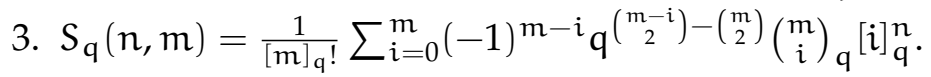

Remark 3.2. We also note that the expression Proposition 3.1 (1) and (3) were obtained in [3, 10, 11, 21, 28]. Proposition 3.1 (2) is due to Carlitz [4, (9)]. It was stated by Gould [13, (3.10)].

From Proposition 3.1 (1), we observe that

$$
\left.\triangle_{\mathrm{q}}^{\mathrm{m}}[x]_{\mathrm{q}}^{\mathrm{n}}\right|_{\mathrm{x}=0}=[\mathrm{m}]_{\mathrm{q}} ! \mathrm{q}\left(\begin{array}{c}
\mathrm{m} \\
2
\end{array}\right) S_{\mathrm{q}}(\mathrm{n}, \mathrm{m})
$$

and

$$
S_{\mathrm{q}}(\mathrm{n}, \mathrm{m})=0 \quad \text { if } \mathrm{n}<\mathrm{m},
$$

since $\left.\triangle_{q}^{m}[x]_{q}^{n}\right|_{x=0}=0$ for $n<m$. Also, by (3.6) and (3.8), if $n=m$, then we get

$$
\left.\triangle_{\mathrm{q}}^{\mathrm{m}}[\mathrm{x}]_{\mathrm{q}}^{\mathrm{m}}\right|_{\mathrm{x}=0}=[\mathrm{m}]_{\mathrm{q}} !_{\mathrm{q}}\left(\begin{array}{c}
\mathrm{m} \\
2
\end{array}\right)
$$

(see [21, p. 107]). This is a q-analog of Euler's result $\triangle^{m} 0^{m}=\triangle_{1}^{m} 0^{m}=m !$, to which it reduces for $q=1$. In fact it is easy to see that

$$
S_{\mathrm{q}}(\mathrm{m}, \mathrm{m})=1
$$

These calculations prove the following result. 
Corollary 3.3. For any positive integers $m$ and $n$, we have

$$
\sum_{i=0}^{m}(-1)^{i} q^{\left(\begin{array}{c}
m-i \\
2
\end{array}\right)}\left(\begin{array}{c}
m \\
i
\end{array}\right)_{q}[i]_{q}^{n}= \begin{cases}0, & \text { if } n<m, \\
(-1)^{m}[m]_{q} !, & \text { if } n=m .\end{cases}
$$

Remark 3.4. If we let $\mathrm{q} \rightarrow 1$ in Corollary 3.3, we obtain Euler's formula in classical analysis (see [12, (2.1)]).

More generally, from (2.3) with $h=1$, the formula for $S_{q}(n, m)$ is intimately connected with the $m$-th $\mathrm{q}$-difference operator $\triangle_{q}^{m}$. Let $f_{q}(x)=[x]_{q}^{n}$ for any nonnegative integer $n$. Then

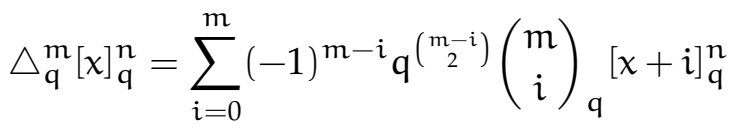

$$
\begin{aligned}
& =\sum_{i=0}^{m}(-1)^{m-i} q^{\left(\begin{array}{c}
m-i \\
2
\end{array}\right)}\left(\begin{array}{c}
m \\
i
\end{array}\right)_{q}\left([x]_{q}+q^{x}[i]_{q}\right)^{n}
\end{aligned}
$$

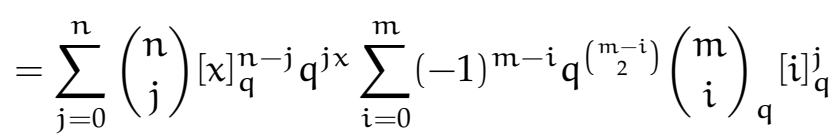

$$
\begin{aligned}
& =\sum_{j=0}^{n}\left(\begin{array}{l}
n \\
j
\end{array}\right)[x]_{q}^{n-j} q^{j x}[m]_{q} ! q\left(\begin{array}{c}
m \\
2
\end{array}\right) S_{q}(j, m) .
\end{aligned}
$$

If $x \rightarrow 0$, (3.10) becomes Proposition 3.1 (1).

Remark 3.5. Many authors investigated q-Stirling numbers in various aspects [3, 10, 11, 26-28]. In [3], Carlitz defined the q-Stirling numbers of the second kind as the numbers $S_{q}(n, m)$ in (3.1). In [10], Corcino et al. defined two forms of q-analogue of noncentral Stirling numbers of the second kind and obtained some properties parallel to those of noncentral Stirling numbers. In [11], Corcino and Montero defined $p$, q-difference operator and obtained an explicit formula analogous to (2.2).

Theorem 3.6. The recurrence for $S_{q}(n, m)$ is given by

$$
S_{q}(n, m)=[-1]_{q}^{n} q^{m n-\left(\begin{array}{c}
m \\
2
\end{array}\right)} \sum_{j=0}^{n}(-1)^{m+n-j}\left(\begin{array}{l}
n \\
j
\end{array}\right)[m]_{1 / q}^{n-j} S_{1 / q}(j, m) .
$$

Proof. Noting that

$$
[m-i]_{q}=[i-m]_{1 / q}[-1]_{q}=q^{m}\left([i]_{1 / q}-[m]_{1 / q}\right)[-1]_{q}, \quad\left(\begin{array}{c}
m \\
i
\end{array}\right)_{q}=q^{i(m-i)}\left(\begin{array}{c}
m \\
i
\end{array}\right)_{1 / q} .
$$

Thus, by (3.11),

$$
\begin{aligned}
& q^{\left(\begin{array}{c}
m \\
2
\end{array}\right)}[m]_{q} ! S_{q}(n, m)=\sum_{i=0}^{m}(-1)^{m-i} q^{\left(\begin{array}{c}
m-i \\
2
\end{array}\right)}\left(\begin{array}{c}
m \\
i
\end{array}\right)_{q}[i]_{q}^{n} \\
& \left.=\sum_{i=0}^{m}(-1)^{i} q^{\left(\begin{array}{c}
i \\
2
\end{array}\right)}\left(\begin{array}{c}
m \\
m-i
\end{array}\right) \underset{q}{q}-i\right]_{q}^{n} \\
& =\sum_{i=0}^{m}(-1)^{i} q^{\left(\begin{array}{c}
i \\
2
\end{array}\right)}\left(\begin{array}{c}
m \\
i
\end{array}\right)_{q}\left(q^{m}\left([i]_{1 / q}-[m]_{1 / q}\right)[-1]_{q}\right)^{n} \\
& =\sum_{i=0}^{m}(-1)^{i} q^{\left(\begin{array}{c}
i \\
2
\end{array}\right) q^{i(m-i)}}\left(\begin{array}{c}
m \\
i
\end{array}\right)_{1 / q} q^{m n} \times[-1]_{q}^{n} \sum_{j=0}^{n}(-1)^{n-j}\left(\begin{array}{c}
n \\
j
\end{array}\right)[i]_{1 / q}^{j}[m]_{1 / q}^{n-j}
\end{aligned}
$$




$$
\left.=[-1]_{q}^{n} q^{m n+\left(\begin{array}{c}
m \\
2
\end{array}\right)} \sum_{j=0}^{n}(-1)^{m+n-j}\left(\begin{array}{c}
n \\
j
\end{array}\right)[m]_{1 / q}^{n-j} \times \sum_{i=0}^{m}(-1)^{m-i}(1 / q)^{(m-i}\right)^{m}\left(\begin{array}{c}
m \\
i
\end{array}\right)_{1 / q}[i]_{1 / q^{\prime}}^{j}
$$

since $\mathrm{q}^{\left(\begin{array}{c}\mathrm{i} \\ 2\end{array}\right)-\mathfrak{i}^{2}+\mathfrak{i m}}=(1 / \mathrm{q})^{\left(\begin{array}{c}\mathrm{m}-\mathrm{i} \\ 2\end{array}\right)} \mathrm{q}^{\left(\begin{array}{c}\mathrm{m} \\ 2\end{array}\right)}$.

From Proposition 3.1 (3) and (3.12) we deduce that

$$
S_{q}(n, m)=[-1]_{q}^{n} q^{m n} \frac{[m]_{1 / q} !}{[m]_{q} !} \sum_{j=0}^{n}(-1)^{m+n-j}\left(\begin{array}{c}
n \\
j
\end{array}\right)[m]_{1 / q}^{n-j} S_{1 / q}(j, m),
$$

whence the theorem follows from $\frac{[m]_{1 / q}}{[m]_{q}}=\frac{1}{q^{m-1}}$.

The following theorem is a direct consequence of equation (3.1).

Theorem 3.7. The q-Stirling numbers $S_{\mathrm{q}}(\mathrm{n}, \mathrm{m})$ are given by

$$
\sum_{j=0}^{n}(-1)^{j} q^{-j}[j]_{q} ! S_{q}(n, j)=\left(-\frac{1}{q}\right)^{n}, \quad \sum_{j=0}^{n}(-1)^{j} q^{-j x}\left(\begin{array}{c}
x+j-1 \\
j
\end{array}\right)_{q}[j]_{q} ! S_{q}(n, j)=\left(-\frac{1}{q}\right)^{n}[x]_{1 / q}^{n} .
$$

Proof. Using (1.4), (3.1) implies that

$$
[x]_{q}^{n}=\sum_{j=0}^{n} q^{\left(\begin{array}{l}
j \\
2
\end{array}\right)}\left(\begin{array}{l}
x \\
j
\end{array}\right)_{q}[j]_{q} ! S_{q}(n, j)
$$

whenever $j$ is a nonnegative integer. The first equation is (3.13) with $x=-1$ while the second equation is (3.13) with $x \rightarrow-x$, since

$$
\begin{aligned}
\left(\begin{array}{c}
-x \\
j
\end{array}\right)_{q} & =\frac{[-x]_{q}[-x-1]_{q} \cdots[-x-j+1]_{q}}{[j]_{q} !} \\
& =\frac{\left(-q^{-x}\right)[x]_{q}\left(-q^{-x-1}\right)[x+1]_{q} \cdots\left(-q^{-x-j+1}\right)[x+j-1]_{q}}{[j]_{q} !} \\
& =(-1)^{j} \frac{1}{q^{j x+\left(\begin{array}{c}
j \\
2
\end{array}\right)}\left(\begin{array}{c}
x+j-1 \\
j
\end{array}\right)_{q} \cdot}
\end{aligned}
$$

This completes the proof.

Corollary 3.8. For any nonnegative integer $n$, we have

$$
\sum_{j=0}^{n}(-1)^{j} q^{-2 j}[j]_{q}[j]_{q} ! S_{q}(n, j)=\left(-\frac{1}{q}\right)^{n}\left([2]_{1 / q}^{n}-1\right) .
$$

Proof. In second equation of Theorem 3.7, let $x=2$. The left hand side, by

$$
\left(\begin{array}{c}
j+1 \\
j
\end{array}\right)_{q}=[j+1]_{q}=q^{j}[1]_{q}+[j]_{q},
$$

becomes

$$
\sum_{j=0}^{n}(-1)^{j} q^{-2 j}\left(q^{j}[1]_{q}+[j]_{q}\right) S_{q}(n, j)=\left(-\frac{1}{q}\right)^{n}[2]_{1 / q}^{n}
$$

and applying first equation of Theorem 3.7 leads to the required result. 
Theorem 3.9. The q-Stirling numbers $S_{\mathrm{q}}(\mathrm{n}, \mathrm{m})$ are given by

$$
\sum_{j=0}^{n}(-1)^{j} q^{-\frac{i}{2}}\left[\frac{1}{2}\right]_{q}^{2 j} \frac{\left([j]_{\left.q^{1 / 2} !\right)^{2}}\right.}{[j]_{q} !}\left(\begin{array}{c}
2 j \\
j
\end{array}\right)_{q^{1 / 2}} S_{q}(n, j)=(-1)^{n} q^{-\frac{n}{2}}\left[\frac{1}{2}\right]_{q}^{n} .
$$

Proof. First, we consider

$$
\begin{aligned}
& {[\mathrm{r}]_{\mathrm{q}}\left[\mathrm{r}-\frac{1}{2}\right]_{\mathrm{q}}[\mathrm{r}-1]_{\mathrm{q}}\left[\mathrm{r}-\frac{3}{2}\right]_{\mathrm{q}} \cdots[\mathrm{r}-\mathrm{k}+1]_{\mathrm{q}}\left[\mathrm{r}-\mathrm{k}+\frac{1}{2}\right]_{\mathrm{q}}} \\
& =[2 r]_{q^{1 / 2}}\left[\frac{1}{2}\right]_{\mathrm{q}}[2 r-1]_{\mathrm{q}^{1 / 2}}\left[\frac{1}{2}\right]_{\mathrm{q}}[2 \mathrm{r}-2]_{\mathrm{q}^{1 / 2}}\left[\frac{1}{2}\right]_{\mathrm{q}}[2 \mathrm{r}-3]_{\mathrm{q}^{1 / 2}}\left[\frac{1}{2}\right]_{\mathrm{q}} \\
& \times \cdots \times[2 r-2 k+2]_{q^{1 / 2}}\left[\frac{1}{2}\right]_{\mathrm{q}}[2 r-2 k+1]_{\mathrm{q}^{1 / 2}}\left[\frac{1}{2}\right]_{\mathrm{q}} \\
& =\left[\frac{1}{2}\right]_{\mathrm{q}}^{2 \mathrm{k}} \frac{[2 \mathrm{r}]_{\mathrm{q}^{1 / 2}} !}{[2 \mathrm{r}-2 \mathrm{k}]_{\mathrm{q}^{1 / 2}} !} \text {. }
\end{aligned}
$$

Multiplying (3.15) by $\frac{1}{\left([k]_{q} !\right)^{2}}$, it is to see that the left hand side of (3.15) can be written as

$$
\frac{[r]_{\mathrm{q}}[r-1]_{\mathrm{q}} \cdots[r-k+1]_{\mathrm{q}}}{[k]_{\mathrm{q}} !} \frac{\left[r-\frac{1}{2}\right]_{\mathrm{q}}\left[\mathrm{r}-\frac{3}{2}\right]_{\mathrm{q}} \cdots\left[r-k+\frac{1}{2}\right]_{\mathrm{q}}}{[k]_{\mathrm{q}} !}=\left(\begin{array}{l}
\mathrm{r} \\
k
\end{array}\right)_{\mathrm{q}}\left(\begin{array}{c}
r-\frac{1}{2} \\
k
\end{array}\right)_{\mathrm{q}}
$$

and the right hand side of (3.15) becomes

$$
\begin{aligned}
& {\left[\frac{1}{2}\right]_{q}^{2 k} \frac{[2 r] q^{1 / 2} !}{[2 r-2 k]_{q^{1 / 2}} !\left([k]_{q^{1 / 2}} !\right)^{2}} \frac{\left([k]_{q^{1 / 2}} !\right)^{2}}{\left([k]_{q} !\right)^{2}}} \\
& =\left[\frac{1}{2}\right]_{q}^{2 k} \frac{[2 r]_{q^{1 / 2}} !}{[2 k]_{q^{1 / 2}} ![2 r-2 k]_{q^{1 / 2}} !} \frac{[2 k]_{q^{1 / 2}} !}{[2 k-k]_{q^{1 / 2}} ![k]_{q^{1 / 2}} !}\left(\frac{[k]_{q^{1 / 2}} !}{[k]_{q} !}\right)^{2} \\
& =\left[\frac{1}{2}\right]_{q}^{2 k}\left(\frac{[k]_{q^{1 / 2}} !}{[k]_{q} !}\right)^{2}\left(\begin{array}{c}
2 r \\
2 k
\end{array}\right)_{q^{1 / 2}}\left(\begin{array}{c}
2 k \\
k
\end{array}\right)_{q^{1 / 2}} \text {. }
\end{aligned}
$$

From (3.16) and (3.17) it is clear that

$$
\left(\begin{array}{l}
r \\
k
\end{array}\right)_{q}\left(\begin{array}{c}
r-\frac{1}{2} \\
k
\end{array}\right)_{q}=\left[\frac{1}{2}\right]_{q}^{2 k}\left(\frac{[k]_{q^{1 / 2} !}}{[k]_{q} !}\right)^{2}\left(\begin{array}{c}
2 r \\
2 k
\end{array}\right)_{q^{1 / 2}}\left(\begin{array}{c}
2 k \\
k
\end{array}\right)_{q^{1 / 2}} .
$$

If we set $k=r=j$ in (3.18), we get

$$
\left(\begin{array}{c}
j-\frac{1}{2} \\
j
\end{array}\right)_{q}=\left[\frac{1}{2}\right]_{q}^{2 j}\left(\frac{[j]_{q^{1 / 2} !}}{[j]_{q} !}\right)^{2}\left(\begin{array}{c}
2 j \\
j
\end{array}\right)_{q^{1 / 2}} .
$$

Similarly, putting $x=\frac{1}{2}$ in (3.14), we get

$$
\left(\begin{array}{c}
j-\frac{1}{2} \\
j
\end{array}\right)_{q}=(-1)^{j} q^{\frac{j^{2}}{2}}\left(\begin{array}{c}
-\frac{1}{2} \\
j
\end{array}\right)_{q} .
$$

Hence, by (3.19) and (3.20),

$$
\left(\begin{array}{c}
-\frac{1}{2} \\
j
\end{array}\right)_{q}=(-1)^{j} q^{-\frac{j^{2}}{2}}\left[\frac{1}{2}\right]_{q}^{2 j}\left(\frac{[j]_{q^{1 / 2} !}}{[j]_{q} !}\right)^{2}\left(\begin{array}{c}
2 j \\
j
\end{array}\right)_{q^{1 / 2}} .
$$


Using (3.21) we substitute $x=-\frac{1}{2}$ in (3.13), obtaining

$$
\sum_{j=0}^{n}(-1)^{j} q^{-\frac{j}{2}}\left[\frac{1}{2}\right]_{q}^{2 j} \frac{\left([j]_{q^{1 / 2}} !\right)^{2}}{[j]_{q} !}\left(\begin{array}{c}
2 j \\
j
\end{array}\right){ }_{q^{1 / 2}} S_{q}(n, j)=(-1)^{n} q^{-\frac{n}{2}}\left[\frac{1}{2}\right]_{q}^{n} .
$$

This completes the proof.

Theorem 3.10. Let $\mathrm{f}_{\mathrm{q}}(\mathrm{x})$ be an arbitrary polynomial of degree $\mathrm{n}$ in $[\mathrm{x}]$, that is, $\mathrm{f}_{\mathrm{q}}(\mathrm{x})=\sum_{\mathrm{k}=0}^{\mathrm{n}} \mathrm{a}_{\mathrm{k}}[\mathrm{x}]_{\mathrm{q}}^{\mathrm{k}}$. Then

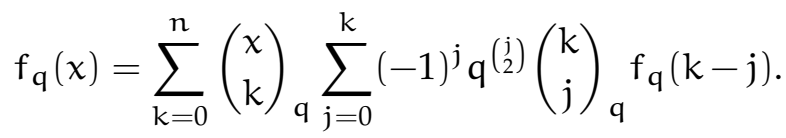

Proof. Since

$$
\sum_{k=0}^{n} \sum_{\alpha=0}^{k} A_{k, \alpha}=\sum_{\alpha=0}^{n} \sum_{k=\alpha}^{n} A_{k, \alpha}
$$

we can apply Proposition 3.1 and (3.13) to obtain

$$
\begin{aligned}
& f_{q}(x)=\sum_{k=0}^{n} a_{k} \sum_{\alpha=0}^{k} q^{\left(\begin{array}{l}
\alpha \\
2
\end{array}\right)}\left(\begin{array}{l}
x \\
\alpha
\end{array}\right)_{q}[\alpha]_{q} ! S_{q}(k, \alpha) \\
& =\sum_{\alpha=0}^{n} q^{\left(\begin{array}{c}
\alpha \\
2
\end{array}\right)}\left(\begin{array}{l}
x \\
\alpha
\end{array}\right)_{q}[\alpha]_{q} ! \sum_{k=\alpha}^{n} a_{k} S_{q}(k, \alpha)
\end{aligned}
$$

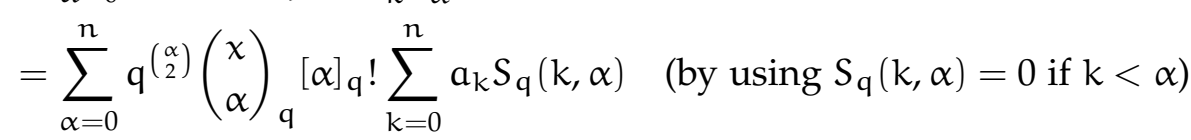

$$
\begin{aligned}
& =\sum_{\alpha=0}^{n}\left(\begin{array}{l}
x \\
\alpha
\end{array}\right) \sum_{\mathrm{q}=0}^{n} a_{k} \sum_{j=0}^{\alpha}(-1)^{j} q^{\left(\begin{array}{l}
j \\
2
\end{array}\right)}\left(\begin{array}{l}
\alpha \\
j
\end{array}\right)_{q}[\alpha-j]_{q}^{k}
\end{aligned}
$$

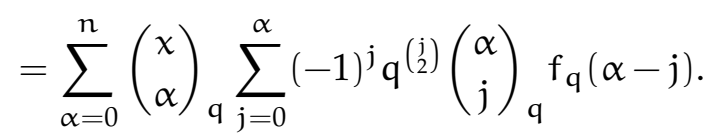

This completes the proof.

Remark 3.11. If $f_{q}(x)=[x]_{q}^{n}$, then Theorem 3.10 becomes (3.13) by using Proposition 3.1 (3). Here are two more examples of Theorem 3.10:

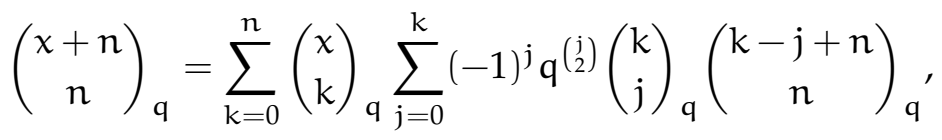

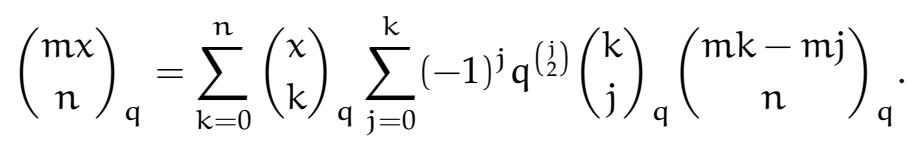

\section{The q-analogue of $\left(x \frac{d}{d x}\right)^{n}$ and its applications}

At the centre is the $q$-derivative operator or Jackson-derivative, here denoted as $D_{q}$ and defined for any polynomial $f$ as follows where $D_{q}$ (q-derivative or Jackson's derivative) is defined by

$$
D_{q} f(x)=\frac{f(q x)-f(x)}{(q-1) x} \text { if } x \neq 0,
$$

and $D_{q} f(x)=f^{\prime}(0)$ if $x=0$, where $q$ is a complex numbers such that $q \neq 0$ and $|q| \neq 1$. It is a simple 
consequence that

$$
D_{\mathrm{q}} x^{\mathrm{n}}=[\mathrm{n}]_{\mathrm{q}} \mathrm{x}^{\mathrm{n}-1}
$$

In the standard approach to the q-calculus two exponential functions are defined by

$$
e_{q}^{x}=\sum_{n=0}^{\infty} \frac{x^{n}}{[n]_{q} !}=\frac{1}{(1-(1-q) x)_{q}^{\infty}} \quad \text { and } \quad E_{q}^{x}=\sum_{n=0}^{\infty} q^{\left(\begin{array}{l}
j \\
2
\end{array}\right)} \frac{x^{n}}{[n]_{q} !}=(1+(1-q) x)_{q}^{\infty} \text {, }
$$

where $q$ is positive, $x$ is complex, and

$$
(1+x)_{q}^{\infty}=\sum_{j=0}^{\infty} q^{\left(\begin{array}{l}
j \\
2
\end{array}\right)} \frac{x^{j}}{(1-q)\left(1-q^{2}\right) \cdots\left(1-q^{j}\right)^{\prime}}, \quad \frac{1}{(1-x)_{q}^{\infty}}=\sum_{j=0}^{\infty} \frac{x^{j}}{(1-q)\left(1-q^{2}\right) \cdots\left(1-q^{j}\right)} .
$$

From (4.2), we easily see that $E_{q}^{x}=e_{1 / q}^{x}$ and

$$
e_{\mathrm{q}}^{\mathrm{x}} \mathrm{E}_{\mathrm{q}}^{-\mathrm{x}}=1
$$

Moreover,

It is known that

$$
D_{q} e_{q}^{x}=e_{q}^{x}, \quad D_{q} E_{q}^{x}=E_{q}^{q x} .
$$

$$
\left(x D_{q}\right)^{n}=\sum_{k=0}^{n} q^{\left(\begin{array}{c}
k \\
2
\end{array}\right)} S_{q}(n, k) x^{k} D_{q}^{k} .
$$

This result was already derived in [20, (76)], [5, Corollary 5.2], and can be shown by a simple example (see also [6, (42)]). Notice that as $q \rightarrow 1$, (4.4) reduces to the well-known operator $\left(x \frac{d}{d x}\right)^{n}$ first thoroughly investigated by Grunert [14] and also by Knopf [18]. That is,

$$
\left(x \frac{d}{d x}\right)^{n}=\sum_{k=0}^{n} x^{k} S(n, k) \frac{d^{k}}{d x^{k}}
$$

Next we consider some expansion of $e_{\mathrm{q}}^{\chi}$. From (4.1), we obtain

$$
\left(x D_{q}\right) e_{q}^{x}=x\left(\frac{[1]_{q}}{[1]_{q} !} x^{0}+\frac{[2]_{q}}{[2]_{q} !} x^{1}+\cdots+\frac{[j]_{q}}{[j]_{q} !} x^{j-1}+\cdots\right)=\sum_{j=1}^{\infty} \frac{[j]_{q}}{[j]_{q} !} x^{j} .
$$

Similarly,

$$
\left(x D_{q}\right)^{n} e_{q}^{x}=\sum_{j=1}^{\infty} \frac{[j]_{q}^{n}}{[j]_{q} !} x^{j}
$$

By (4.3) and (4.4), we may write

$$
\left(x D_{q}\right)^{n} e_{q}^{x}=\sum_{k=0}^{n} q^{\left(\begin{array}{c}
k \\
2
\end{array}\right)} S_{q}(n, k) x^{k} D_{q}^{k} e_{q}^{x}=e_{q}^{x} \sum_{k=0}^{n} q^{\left(\begin{array}{c}
k \\
2
\end{array}\right)} S_{q}(n, k) x^{k},
$$

so that, using (4.5),

$$
e_{\mathrm{q}}^{x} \sum_{k=0}^{n} q^{\left(\begin{array}{l}
k \\
2
\end{array}\right)} S_{q}(n, k) x^{k}=\sum_{j=1}^{\infty} \frac{[j]_{q}^{n}}{[j]_{q} !} x^{j} .
$$

Multiplying both sides of (4.6) by $\mathrm{E}_{\mathrm{q}}^{-\mathrm{x}}$ and substituting the series for $\mathrm{E}_{\mathrm{q}}^{-\mathrm{x}}$ gives

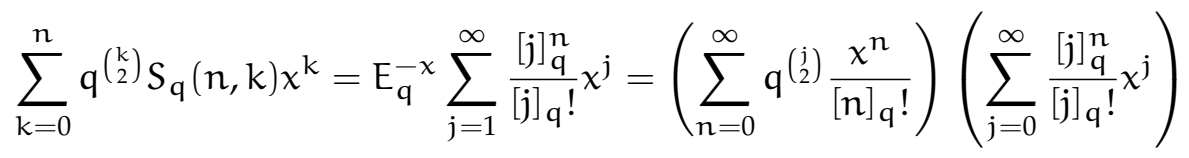

$$
\begin{aligned}
& =\sum_{k=0}^{\infty}\left(\frac{1}{[k]_{q} !} \sum_{i=0}^{k}(-1)^{i} q^{\left(\begin{array}{c}
i \\
2
\end{array}\right)}\left(\begin{array}{l}
k \\
i
\end{array}\right)_{q}[k-i]_{q}^{n}\right) x^{k} \text {. }
\end{aligned}
$$

Equating corresponding coefficients of $x^{k}$ in (4.7) for $k=0,1, \ldots, n$ gives 


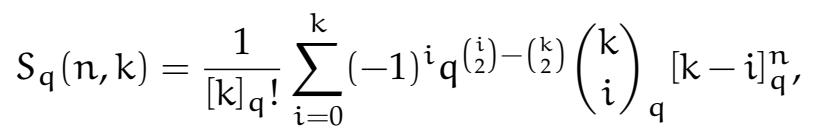

which is equivalent to Proposition 3.1 (3).

Letting $f_{n, r}(x)=\sum_{k=0}^{n} q^{k}[k]_{q}^{r} x^{k}$, by (4.1) it is immediate that

$$
\left(x D_{q}\right) f_{n, r}(x)=\sum_{k=0}^{n} q^{k}[k]_{q}^{r+1} x^{k}=f_{n, r+1}(x),
$$

and that, by induction on $\ell$,

$$
\left(x D_{q}\right)^{\ell} f_{n, r}(x)=\sum_{k=0}^{n} q^{k}[k]_{q}^{r+\ell} x^{k}=f_{n, r+\ell}(x) .
$$

If we let $r=0$, it is obvious that

$$
f_{n, \ell}(x)=\left(x D_{q}\right)^{\ell} f_{n, 0}(x)=\left(x D_{q}\right)^{\ell}\left(\sum_{i=0}^{n} q^{i} x^{i}\right) .
$$

By (4.1) and (4.4) we see that

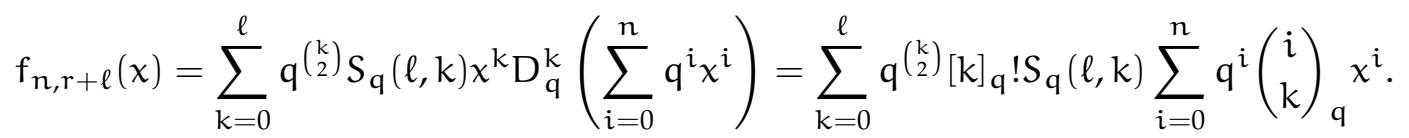

If $x=1,(4.8)$ becomes

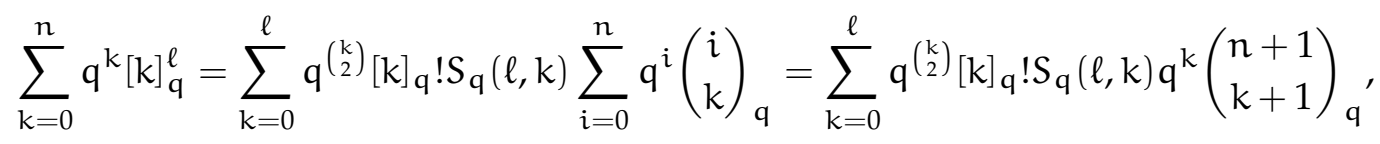

where the last equality in (4.9) follows from the q-analogues of the Hockey Stick identities [9, p. 7]:

$$
\left(\begin{array}{l}
n+1 \\
k+1
\end{array}\right)_{q}=\sum_{i=k}^{n} q^{i-k}\left(\begin{array}{l}
i \\
k
\end{array}\right)_{q}=\sum_{i=0}^{n} q^{i-k}\left(\begin{array}{l}
i \\
k
\end{array}\right)_{q}
$$

Note that (4.9) is equivalent to (6.1) in [3, p. 994].

\section{Acknowledgment}

The authors are enormously grateful to the anonymous referees whose comments and suggestions lead to a large improvement of the paper.

\section{References}

[1] K. N. Boyadzhiev, Close encounters with the Stirling numbers of the second kind, Math. Mag., 85 (2012), 252-266. 1, 2.2

[2] Y. Cai, M. A. Readdy, q-Stirling numbers: a new view, Adv. Appl. Math., 86 (2017), 50-80. 3

[3] L. Carlitz, q-Bernoulli numbers and polynomials, Duke Math. J., 15 (1948), 987-1000. 1, 1, 1, 2, 2, 3, 3, 3.2, 3.5, 4

[4] L. Carlitz, On abelian fields, Trans. Amer. Math. Soc., 35 (1933), 122-136. 1, 3.2

[5] C. A. Charalambides, On the q-differences of the generalized q-factorials, J. Statist. Plann. Inference, 54 (1996), 31-43. 4

[6] J. Cigler, Operatormethoden fur q-Identitaten (German), Monatsh. Math., 88 (1979), 87-105. 1, 4

[7] L. Comtet, Advanced Combinatorics, Springer Netherlands, Dordrecht, (1974). 1

[8] K. Conrad, A q-analogue of Mahler expansions I, Adv. Math., 153 (2000), 185-230. 2 
[9] R. B. Corcino, On p, q-binomial coefficients, Integers, 8 (2008), 16 pages. 4

[10] C. B. Corcino, R. B. Corcino, J. M. Ontolan, C. M. Perez-Fernandez, E. R. Cantallopez, The Hankel transform of q-noncentral Bell numbers, Int. J. Math. Math. Sci., 2015 (2015), 10 pages. 3.2, 3.5

[11] B. R. Corcino, C. B. Montero, On p, q-difference operator, J. Korean Math. Soc., 49 (2012), 537-547. $3.2,3.5$

[12] H. W. Gould, Euler's formula for nth differences of powers, Amer. Math. Monthly, 85 (1978), 450-467. 1, 1, 3.4

[13] H. W. Gould, The q-Stirling numbers of first and second kinds, Duke Math. J., 28 (1961), 281-289. 1, 1, 3, 3.2

[14] J. A. Grunert, Uber die Summerung der Reihen von der Form $A \phi(0), \quad A_{1} \phi(1) x, \quad A_{2} \phi(2) x^{2}, \ldots A_{n} \phi(n) x^{n}, \ldots$, wo A eine beliebige constante Gre, $A_{n}$ eine beliebige und $\phi(n)$ eine ganze rationale algebraische Function der positiven ganzen Zahl n bezeichnet, J. Reine Angew. Math., 25 (1843), 240-279. 4

[15] F. H. Jackson, On q-functions and a certain difference operator, Trans. Roy. Soc. Edinburgh, 46 (1909), 253-281. 2

[16] C. Jordan, Calculus of finite differences, Chelsea, New York, (1950). 1

[17] M.-S. Kim, J.-W. Son, A note on q-difference operators, Commun. Korean Math. Soc., 17 (2002), 423-430. 2, 2, 2

[18] P. M. Knopf, The Operator $\left(x \frac{d}{d x}\right)^{n}$ and Its Applications to Series, Math. Mag., 76 (2003), 364-371. 4

[19] I. Kucukoglu, A. Bayad, Y. Simsek, k-ary Lyndon words and necklaces arising as rational arguments of Hurwitz-Lerch zeta function and Apostol-Bernoulli polynomials, Mediterr. J. Math., 14 (2017), 16 pages. 1

[20] T. Mansour, M. Schork, M. Shattuck, The Generalized Stirling and Bell Numbers Revisited, J. Integer Seq., 15 (2012), 47 pp. 4

[21] S. Milne, A q-analogue of restricted growth functions, Dobinski's equality, and Charlier polynomials, Trans. Amer. Math. Soc., 245 (1978), 89-118. 1, 1, 3, 3.2, 3

[22] H. Ozden, I. N. Cangul, Y. Simsek, Generalized q-Stirling numbers and their interpolation functions, Axioms, 2 (2013), 10-19. 1

[23] B. E. Sagan, Congruence properties of q-analogs, Adv. Math., 95 (1992), 127-143. 1

[24] Y. Simsek, Combinatorial inequalities and sums involving Bernstein polynomials and basis functions, J. Inequal. Spec. Funct., 8 (2017), 15-24. 1

[25] Y. Simsek, Generating functions for generalized Stirling type numbers, Array type polynomials, Eulerian type polynomials and their applications, Fixed Point Theory Appl., 2013 (2013), 28 pages. 1

[26] Y. Simsek, On q-deformed Stirling numbers, Int. J. Math. Comput., 15 (2012), 70-80. 3.5

[27] Y. Simsek, A. Bayad, V. Lokesha, q-Bernstein polynomials related to q-Frobenius-Euler polynomials, l-functions, and q-Stirling numbers, Math. Methods Appl. Sci., 35 (2012), 877-884.

[28] S.-Z. Song, G.-S. Cheon, Y.-B. Jun, L. B. Beasley, A q-analogue of the generalized factorial numbers, J. Korean Math. Soc., 47 (2010), 645-657. 3.2, 3.5

[29] M. Wachs, D. White, p, q-Stirling numbers and set partition statistics, J. Combin. Theory Ser. A, 56 (1991), $27-46.1$

[30] M. Ward, A calculus of sequences, Amer. J. Math., 58 (1936), 255-266. 1

[31] J. Zeng, The q-Stirling numbers, continued fractions and the q-Charlier and q-Laguerre polynomials, J. Comput. Appl. Math., 57 (1995), 413-424. 1 